\title{
Production of Optimized Layered Products USING INTELLIGENT SUPPORT
}

\author{
Ahmet Cekic ${ }^{\mathrm{a}}$, Nebojsa Rasovic ${ }^{\mathrm{b}}$, Milenko Obad ${ }^{\mathrm{b}}$, Jasmin Kaljun $^{\mathrm{c}}$, \\ Bojan Dolsak ${ }^{\mathrm{c}}$, Derzija Begic-Hajdarevic ${ }^{\mathrm{a}}$
}

\author{
${ }^{a}$ Faculty of Mechanical Engineering, University of Sarajevo, Vilsonovo setaliste 9, Sarajevo 71000, \\ Bosnia and Herzegovina \\ ${ }^{b}$ Faculty of Mechanical Engineering and Computing, University of Mostar, Matice hrvatske bb, Mostar 88000 , \\ Bosnia and Herzegovina \\ ${ }^{c}$ Faculty of Mechanical Engineering, University of Maribor, Smetanova 17, Maribor 2000, Slovenia
}

\begin{abstract}
Layered manufacturing technology allows rapid production of parts, which can be used for various engineering evaluation and analyses. For that reason, there is a need for developing an expert tool to fully implemented analyses of a CAD model at the some phases of design process. This paper introduces multi criteria decision based on intelligent advisory system. Knowledge base contains collected experts recommendations from functional, aesthetic and ergonomic field. These recommendations are improving model characteristics for its better exploitation. The ultimate goal of this expert tool is to provide the highest level of advice, depending on the limitations, such as surface quality, time production, manufacturing, assembling, or geometrical complexity. This model system should be applicable for the most CAD models. A case study with the electrical switch is carried out in order to show usefulness of application for specific problems in the layered manufacturing process in order to get better final part. The results obtained from a case study are implemented through 3D Print Technology.
\end{abstract}

Keyword: adaptive slicing; build orientation; expert systems
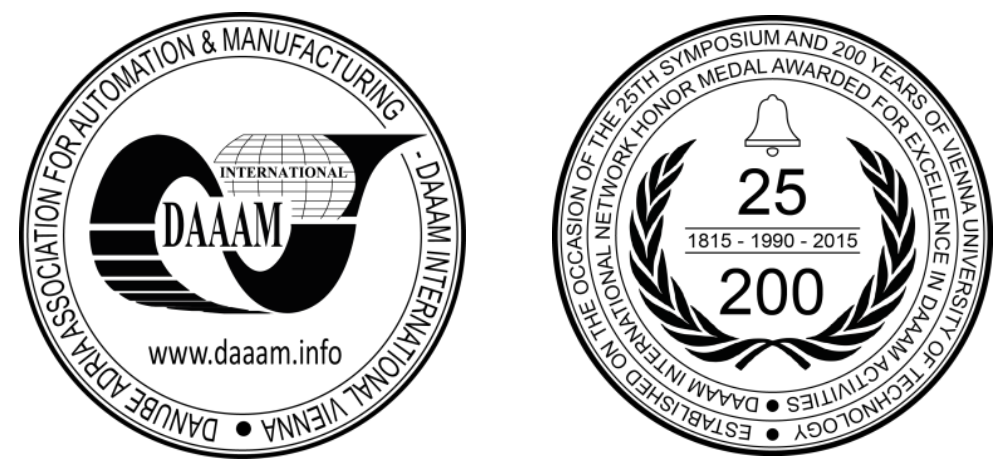

This Publication has to be referred as: Cekic, A[hmet]; Rasovic, N[ebojsa]; Obad, M[ilenko]; Kaljun, J[asmin]; Dolsek, B[ojan] \& Begic-Hajdarevic, D[erzija] (2016). Production of Optimized Layered Products using Intelligent Support, Proceedings of the 26th DAAAM International Symposium, pp.0271-0279, B. Katalinic (Ed.), Published by DAAAM International, ISBN 978-3-902734-07-5, ISSN 1726-9679, Vienna, Austria DOI: $10.2507 / 26$ th.daaam.proceedings.037 


\section{Introduction}

The layered manufacturing technology is widely used in modern industry all over the world. It allows rapid production of any object's form in all design phases. Parts obtained by this technology go not only for visual presentations, but also in real function. Because of its wide availability, there are a lot of people, designers and engineers who use and control some of the layered manufacturing methods. Mostly, the layered methods have a common way of production, but some differences between them make great potential for manipulation of a virtual model. These differences can also make final product more expensive, which does not favour in exploiting to the market. The layered manufacturing is basically a different manufacturing process, where the parts are obtained from the CAD model to the direct numerical control $[1,2]$. This involves a successive addition of the main material in the form of two-dimensional layers to get solid pre-defined shape. A distinct advantage of the parts gotten by a technique of layer-by-layer is that the geometric complexity of the parts has smaller impact on the production process than in case of conventional production processes. The whole production process is based on creating a 3D computer model by solid modelling of the certain product. This step represents an input in slicing process and obtaining a series of 2D layers. These layers are information for layered manufacturing systems and prepare machine for production. Final step including post-processing operations $[3,4]$.

A lot of additive technologies have a possibility for large oscillation in the layer thickness. The reason is that the thicker layers leading to the faster production but also less accuracy [5]. This is not a problem in some cases where important to produce parts in a short time interval. The parts orientation inside of a machine have a great impact on its accuracy [6]. There are a lot of cases where the parts have a complex geometry in direction of multi axes and therefore an orientation may not be ideal for each surface area [7]. Besides, it is very important to preserve the geometry precision of certain features in comparison with one to each other, so that the correct orientation is crucial. Also, the key orientation can be in conflict with some other criteria such as time production or support structure [8].

The main goal of this paper is creating an intelligent decision support in design process of products to be produced by the layered manufacturing methods. The starting point of the intelligent support is a CAD model observing through analyses of multiple characteristics that define overall design of the final product. First of all, it is necessary to collect and apply systematic approach to edit knowledge in the field of product design and the layered manufacturing. Upon this, it makes a formal entry of the collected knowledge for creating intelligent advisory system which on the basis of input parameters help in faster and better decisions [9]. The parts quality involve satisfaction of all characteristics such as shape, compliance and function [10].

\section{Problems and constraints in the layered manufacturing process}

The key step in the layered manufacturing process is slicing of a 3D model in 2D contours. STL format shows a model by approximating its spatial surfaces in a number of small triangles planes (Error! Reference source not found.a), which significantly simplify the slicing process, but also introduce unavoidable problems concerning the precision. Final products most often have a very noticeable staircase effect (Fig. 1b). A boundary of the CAD model is marked with blue line, and the part produced by layered method is marked with black line. It clears that there is a difference between boundary of the CAD model and the model obtained by each single layer. A calculated layer thickness has to be between minimal and maximal value which are defined by the characteristics of the layered manufacturing system.

A specific product designs, considering build problems and constraints, need to be carefully analyzed in order to achieve optimal parameters for production process. For all these reasons, there is a need for introducing the intelligent decision support in design process of products to be produced by the layered manufacturing methods. In that circumstances, it has been developed an intelligent prototype module to reduce product development of both time and costs. This intelligent prototype is called Products Design.

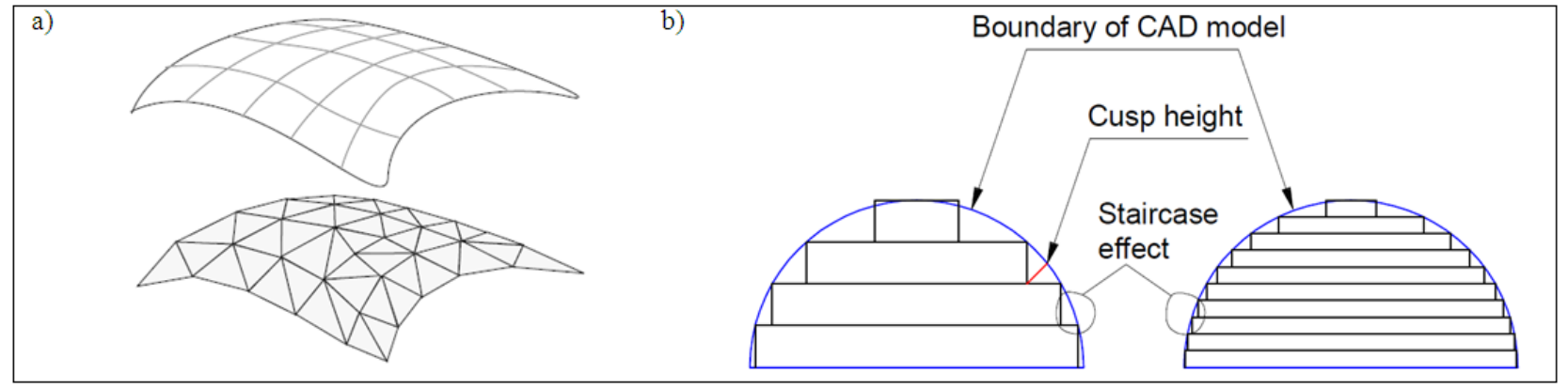

Fig. 1. a) STL approximation of the curved surfaces; b) staircase effect [4] 


\section{Development of the intelligent decision support}

The difficulties that arise in a process of concept evaluation are those of initial design, so that the process is limited by both knowledge and information. In such circumstances, it is crucial to develop a mechanism for concept selection and improvement, or therefore variants that shell ultimately improve the product. This can be achieved by mutual comparison and evaluation of several variants that are defined by a set of criteria. The results are leading to continuous technique repetition in the iterations until the designer is satisfied with results. The evaluation process takes three steps [11]:

- Criteria selection for comparison,

- Variants selection for comparison,

- Marks generation.

The above mentioned approach of treating of products design through mathematical model of weighting factors is used as both starting point and foundation for development of the intelligent support module in the layered manufacturing process. In the view of the specific values of individual zones, a design of the CAD model has to be treated through the certain features that are specific, in order to achieve optimal parameters, where the limits of these zones are defined by features area [12]. This aims at the ability to define different optimal thicknesses in the zones. It is essential to use an adaptive slicing method in order to determine every single layer of the total object height. Weighting factors of the surface (W) are related to the layer thickness control, which ultimately defines the issue of surface quality and other specifications. The procedure of defining weighting factors may be different taking into account the complexity of the CAD models from the criteria aspect for these models. A matrix decision method was used for the weighting factors calculations.

\subsection{The weighting factors calculation by mathematical method of the matrix decision}

It should be highlighted that design for manufacturing requirements are reflected in the fulfillment of tasks in terms of:

- Technical requirements of the model (function, form, fit, production, assembly...),

- Aesthetic requirements of the model (visualization),

- Ergonomic requirements of the model (ergonomic design).

The recognized requirements as model features become criteria for application of a mathematical model of weighting factors, which are used for evaluation these features. A model of the car's gearshift, shown in the Fig. 2, was used for the purpose of weighting factors calculations.

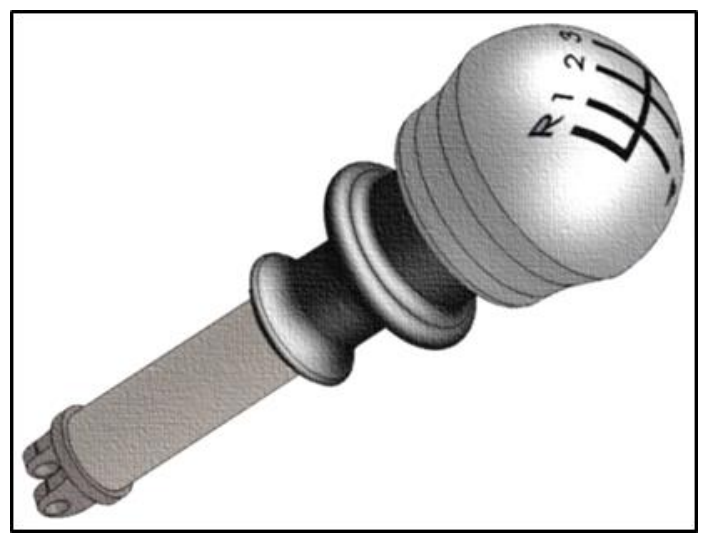

Fig. 2. Design of the product in function of the tasks performing

By detailed approach in the analysis of the model (Fig. 3), it was identified three areas of the model which is specific to some manufacturing demands. The first zone is a part of the joint that connects the handle to the rest of the gearbox. It allows connection and smooth work of shifting operations, and he is very important part in order to its expediency in terms of functionality. Since it is a hinged gear, requires the accurate level of manufacturing. The central zone of the model has a cylindrical shape and linking the two zones of the model and besides aesthetic importance does not have other features. As such, there are no special requirements in terms of accurate manufacturing. The third zone of the model makes handrail, which is in frequent contact with the palm of the driver's hand, and due to its primary purpose an ergonomic importance is given. The parameters of the manufacturing process may not have a high priority of accurate level (Fig. 3). 


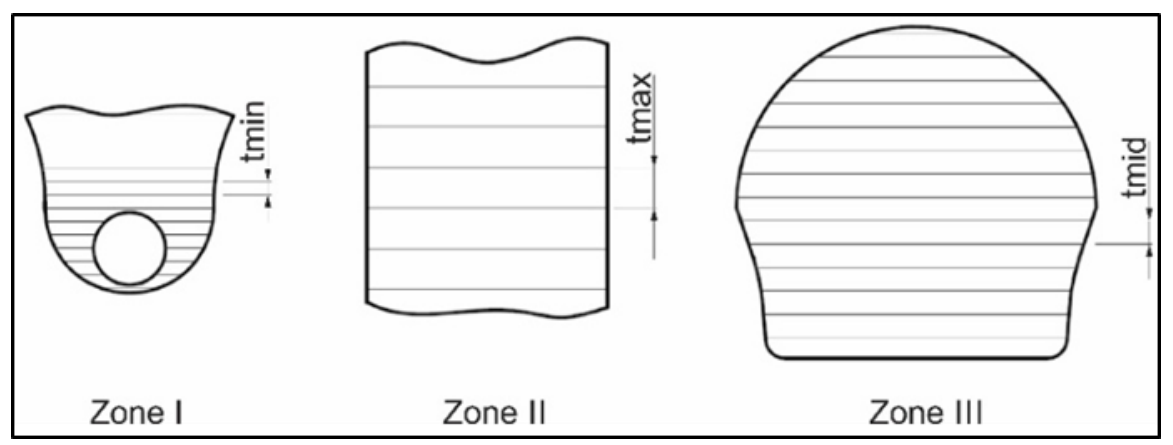

Fig. 3. Specific zones of the gearshift model

By using the method of matrix decision [11], gearshift model includes some criteria which are related to the geometry precision in the zone with functional tasks, aesthetics as visual impression of the surfaces and ergonomics feeling of the surfaces. Each of these criteria have certain weighting factors as follows:

- Geometry $-45 \%$,

- Aesthetic $-25 \%$,

- Ergonomic $-30 \%$.

A sum of the values of the weighting factors (W.F.) have to be up to $100 \%$. Each variant area is assigned value of the rating factor (R.F.) depending (Equation 1) on the importance of each criteria (Table 1) [11].

$W . R . F=W . F . x R . F$.

\begin{tabular}{|c|c|c|c|c|}
\hline Designed & Geometric & Aesthetic & Ergonomic & \multirow{2}{*}{ Sum } \\
\hline & \multicolumn{3}{|c|}{ Weighting factors } & \\
\hline $\begin{array}{l}\text { Variant } \\
\text { area }\end{array}$ & 0,45 & 0,25 & 0,3 & $\sum \mathrm{W} . \mathrm{F} .=1$ \\
\hline Zone I & 0,675 & 0,5 & 0,5 & $\sum$ W.R.F. $=0,95$ \\
\hline Zone II & 0,5 & 0,25 & 0,5 & $\sum$ W.R.F. $=0,625$ \\
\hline Zone III & 0,225 & 0,125 & 0,45 & $\sum$ W.R.F. $=0,8$ \\
\hline
\end{tabular}

Table 1. The values of the weighting factors by using mathematical method of the matrix decision [9]

$\mathrm{T}$ his way of the weighting factors calculation can lead to the fact that the model zones require a separate treatment in determining of the process parameters. These values have to be joined to overall layer thickness. If the new value of the layer thickness exceeds a minimal or maximal allowable height, it means that new value has to be corrected at the boundary value. The Table 1 shows that the corresponding value of the weighting factor has to have big impact on the final layer thickness in the zone II (Equations 2 - 4) [13, 14].

Zone I : $t_{\text {final }}=\frac{t}{W_{I}}=\frac{t}{0,95}\left\{t_{\min } \leq t_{\text {final }} \leq t_{\max }\right\}$

Zone I : $t_{\text {final }}=\frac{t}{W_{I I}}=\frac{t}{0,625}\left\{t_{\min } \leq t_{\text {final }} \leq t_{\max }\right\}$

Zone I: $t_{\text {final }}=\frac{t}{W_{I I I}}=\frac{t}{0,8}\left\{t_{\min } \leq t_{\text {final }} \leq t_{\max }\right\}$

With this approach, there has been a compromise between diametrically opposed demands included in the model as well. At the one side, this was accomplished by achieving satisfactory quality of desired surface, but at the other side there is the time required to obtain the product. An additional advantage of this approach stems from the fact 
that it is adaptive process planning, so that the complexity of three-dimensional model is analyzed by its twodimensional display.

\subsection{Building knowledge base}

Intelligent decision support for the layered manufacturing process is based on the series of rules, which define the procedure for building process. In the expert system technology, each of the expert's rules is heuristic. The combination of all heuristics allows making an overall decision problem to be solved. Main functional, aesthetic or ergonomic features of some CAD model are incorporated in the intelligent module and they present influential parameters in process decision (Fig. 4). First of all, all crucial issues about building process lead out in two steps. The first step is an examination of the importance of the function through a detailed analysis of the geometry. In the second step, the collected knowledge is organized and written in the form of production rules in order to be used by the intelligent system.

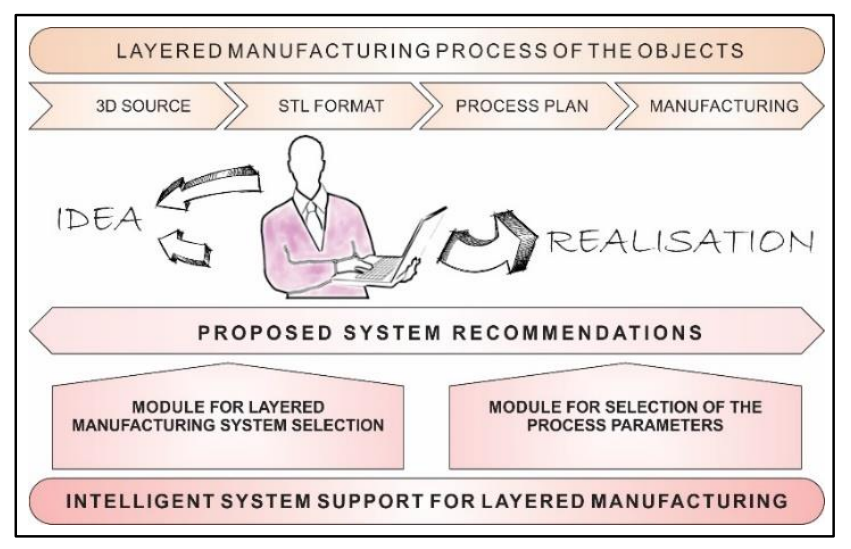

Fig. 4. Product development cycle using intelligent advisory system

Different classes are interconnected by various attributes and their values at the head of the rules in order to describe case specific situations, in which those recommendations for layer thickness, as listed in the body of the rules, should be taken into consideration as for example:

\section{IF:} THEN:

The model surfaces need to be produced with high accuracy

Layer thickness has to be minimal.

\section{Case study}

A case study dealing with the design process of products to be produced by the layered manufacturing methods. For implementation of a case study, the electrical switch (Fig. 5) is used, in order to demonstrate practical application of the knowledge base of the expert system named Products Designed. The goal is to get parameters from the expert system in a form of advice in order to improve the production parts quality. Taking into account that each object builds layer-by-layer method, selection of build orientation is very important issue and it presents essential factor which effects on the final product. In order to increase accuracy in some model areas, the geometric features need to be carefully analyzed. Because of that, proper selection can visibly improve final surfaces and contribute to the entire accuracy of a final part. Each of these features provide orientation condition.

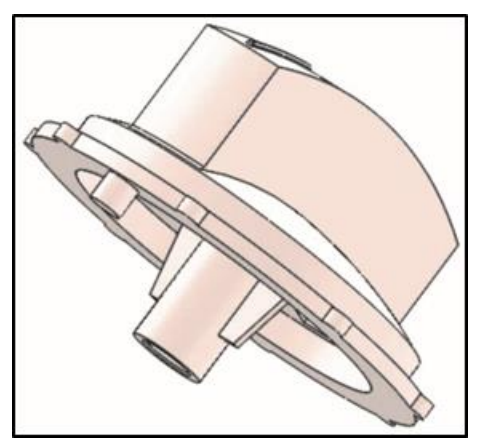

Fig. 5. The electrical switch 
The electrical switch carries various geometric features and for its implementation, system is applied in order to generate some recommendations on behalf better production. The intelligent system in a decision making offers possible solutions (recommendations) based on the set of conditions in a few individual steps. The quality and usefulness of advice provided by the intelligent system depends on the qualities of the input parameters. They present conditions for articulation function tasks. For a closer look, a type of the analysis that was conducted over the model is functional.

INFERENCE RESULTS:

(1) Build orientation along $\mathrm{Z}$ axis direction: Conf $=30.0$

(2) Build orientation along $X$ axis direction: Conf $=20.0$

(1) Features axes of the highest values are parallel with $\mathrm{Z}$ axis of the machine!

(2) Features axes of the lowest values are perpendicular on the $\mathrm{Z}$ axis of the machine!

Consider layer thickness with respect to required production influential criterion.

Reduce height of the support structure at the minimum.

\section{Products D E S I G N \\ Intelligent Decision Support Module \\ for design process of products \\ to be produced by layered manufacturing}

Fig. 6. The results by using the system-analysis of the geometrical complexity

To obtain outline size of entire object, the user is prompted by advisory system to select approximate model volume, which is the most similar to the one of the basic volumes. This fact is important from the viewpoint of the build time. If the object is too long, the user is in situation to lie down object in the build chamber. In the next stages, system collects data about model geometry. To achieve this objective, the optimum value is computed from the confidence function. Therefore, confidence variables are used to calculate an overall probability value for whatever the variable represents. Knowledge base contains many possibilities which the user must rank. There are based on the considering of parameters. From the results display (Fig. 6), the system is proposing the option one (1), because the value of the first option is higher than the second option. Total sum is obtained from particular conditions, derived from geometric features. That means that the object needs to be aligned, so that the features axes of the highest values are parallel with $\mathrm{Z}$ axis of the machine (Fig. 7).

\section{The electrical switch}
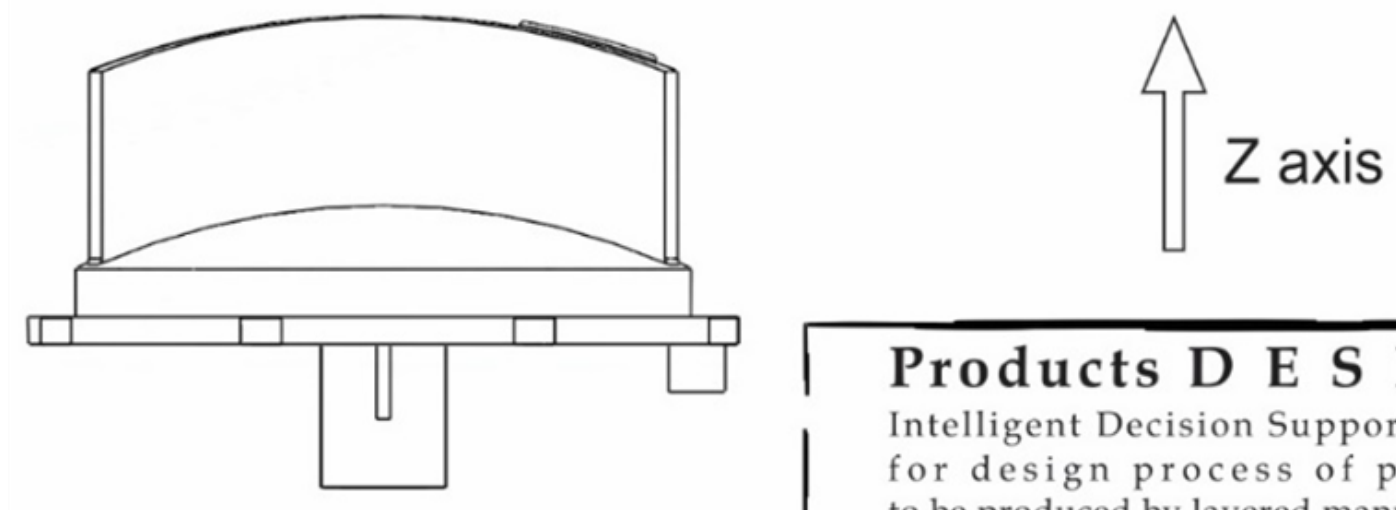

\section{Products D E S I G N}

Intelligent Decision Support Module for design process of products to be produced by layered manufacturing

Fig. 7. Graphic solution of the offered recommendations 
Quality control is a key factor in modern approach of the products design process. The ability to provide the geometry from its original and making better transition from vectors formats to physical model are major challenge in modern engineering. In order to verify produced shape and its fit and function within some assembly, the most designers are implementing the various measuring techniques. Optical 3D scanning, based on the principle of fringe projection, has proven to be both an accurate and cost-effective alternative delivering more dimensional information to aid in quality control and process optimization by creating more meaningful measuring reports. The mobile ATOS 3D optical scanner has a unique capability of being user-configurable to address various measuring requirements [15]. Scanner is supplied by the geometric data from the existing physical object. Thus obtained the geometric data are used by analyze the accuracy of the object surfaces on the electrical switch (Fig. 6). For successful scanning, it is necessary to implement right referent marking on the surfaces of the physical object. All measurements are automatically merged into one, using the referent markers to achieve a common global coordinate system. To perform a comparison of scanned physical and CAD source data, the scanned data are usually converted into CAD data coordinate position. For comparison purposes, the data is entered into GOM Inspect Software, where the aligned models are compared. With this type of alignment, the system requires from user to define the nominal point first, at the CAD model, but then to select actual point at the scanned model. Based on defined reference points, the system calculates the best fit for the actual coordinates and suggests a possible alignment (Fig. 8).

In order to visualize surfaces of the models, colour to each particular part of the objects are added (Fig. 9). This process creates a colour map for easier interpretation and transferring dimensional precision of the produced models which are compared by the CAD model. Reddish areas on the map indicate that the scanned surfaces of the test pieces are over measured dimensions in relate to the surfaces of the CAD model. In contrast, the blue areas on the map indicate that the scanned surfaces of the test pieces are measured according to the surfaces of the CAD model. It automatically displays colourful deviation scale, which is based on the precisely calculated deviation values. Deviation scale can also be finely adjusted or adapted by the user.

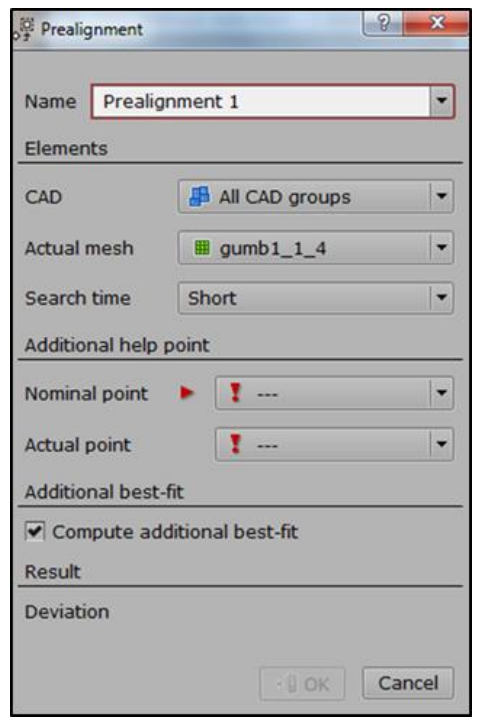

Fig. 8. Pre-alignment method of the models adjust to be compared

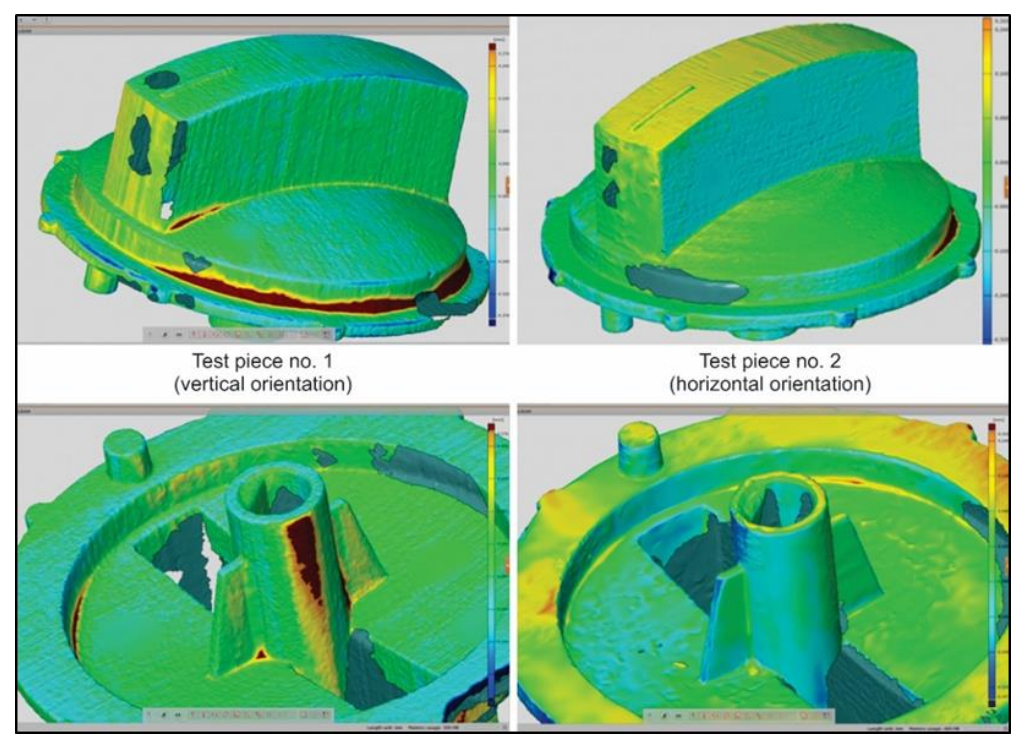

Fig. 9. Surface deviation of the scanned models which are compared by the CAD model 
Based on comparison method, it can see clear differences in surfaces precision of the two test pieces (Fig. 9). The first thing that attracts attention is certainly a cylindrical hole that passes through the central part of the model volume. In vertical orientation, colour map highlights the extremely red colour, which gives a clear picture of the deviation of the part and that is the largest one and is around 0,4 mm. Taking into account the model produced in horizontal orientation, deviation value in that area is around $-0,1 \mathrm{~mm}$, which is visible by ordinary observation. The handle area has surface with high level of staircase effect, which is evident on the model in vertical orientation.

According to the analysis, it is concluded that the model built in the horizontal orientation has more precise geometry than the model built in vertical direction. Also, stair stepping effect has smaller impact on model built in horizontal orientation. The results which are obtained by conducted case study confirming recommendations provided by expert system.

\section{Conclusion}

An important task of design process is based on the fact that shell simultaneously reduce production costs and increase production quality of the model part. Design process is combination of series of phases. Each phase contains a set of design guidelines in order to improve model part. The main benefit of application of additive process which is integrated in the product design process is the ability to create extremely complex shapes and geometric features. A possibility of the parts building in any phase of design process is great emphasis for engineers in order to conducting various analyses of the product development. Limitations which are imposed in design process and its implementation are virtual limitations. Thus, everything can be modelled and created in the virtual world first, then it can be realized in the real world.

In that context, knowledge relating to the design process of products to be produced by layered manufacturing method was collected and organised in the form of production rules, which are the closest with the human's way of making decisions. The knowledge built in the model of the intelligent system named Products Design is structured in the form of different classes interconnected by various attributes and their values. An user may expect certain recommendations by the results provided by intelligent system, which are useful in various phases of a design process.

It could be summarised on the basis of the case study that these recommendation lead out to the improved final product as the result of the conducted design process. The layered manufacturing is not only production, but also it includes a whole process from idea to the realisation. That process includes some iterations in order to develop comprehensive attributes of the model.

The goal of the future researches will be defining a framework of the methodology by which the engineers would be able to successfully recreate surfaces that are defined by designers. Recreation will be mathematically resulted as the simplified surface that is parametrically recorded. At the same time, engineers will also have the opportunity to use recommendations regarding preparation of the production process with layered technologies which will result in technically, strength and aesthetically acceptable product.

\section{Acknowledgements}

The case study presented in this paper was conducted in cooperation with Faculty of Mechanical Engineering of University of Maribor.

\section{References}

[1] Gibson, I.; Rosen, D. W.; Stucker, B. Additive manufacturing technologies; rapid prototyping to direct digital manufacturing. New York: Springer, 2010.

[2] Aburaia, M.; Markl, E.; Stuja, K. New Concept for Design and Control of 4 Axis Robot Using the Additive Manufacturing Technology. Procedia Enginering 100 (2015), pp. 1364-1369.

[3] Udroiu, R.; Nedelcu, A. Optimization of Additive Manufacturing Processes Focused on 3D Printing. Available: http://www.intechopen.com/articles/show/title/optimization-of-additive-manufacturing-processes-focused-on-3dprinting, (2011).

[4] Paulic, M.; Irgolic, T.; Balic, J.; Cus, F.; Cupar, A.; Brajlih,T.; Drstvensek, I. Reverse of Parts with Optical Scanning and Additive Manufacturing. Procedia Enginering 69 (2014), pp. 795-803.

[5] Chen, Y.; Lu, J. RP part surface quality versus build orientation: when the layers are getting thinner. // The International Journal of Advanced Manufacturing Technology, (2012), pp. 1-9.

[6] Rianmora, S.; Koomsap, P. Recommended slicing positions for adaptive direct slicing by image processing technique. // The International Journal of Advanced Manufacturing Technology. 46, 9-12 (2010), pp. 1021-1033.

[7] Canellidis, V.; Giannatsis, J.; Dedoussis, V. Genetic-algorithm-based multi-objective optimization of the build orientation in stereolithography. // The International Journal of Advanced Manufacturing Technology. 45, 7-8 (2009), pp. 714-730.

[8] Byun, H.S.; Lee, K. H. Determination of the optimal build direction for different rapid prototyping processes using multi-criterion decision making. // Robotics and Computer-Integrated Manufacturing. 22, 1 (2006), pp. 69-80. 
[9] Kaljun, J.; Dolsak, B. Ergonomic design knowledge built in the intelligent decision support system. // International Journal of Industrial Ergonomics. 42, 1 (2012), pp. 162-171.

[10] Dolsak, B.; Novak, M. Intelligent decision support for structural design analysis. // Advanced Engineering Informatics. 25, 2 (2011), pp. 330-340.

[11] Haik, Y. Engineering design process. [South Melbourne, Victoria], Australia; Pacific Grove, CA: Thomson/Brooks/Cole, 2003.

[12] Rasovic, N.; Kaljun, J.; Obad, M. Intelligent decision support system for adaptive slicing in RPT process, in Proceedings of The 23rd International Symposium, Zadar, Croatia, 2012, pp. 1095-1098.

[13] Rasovic, N.; Obad, M., "Adaptive slicing in 3D printing process, In Machine And Industrial Design In Mechanical Engineering KOD 2012, Balatonfüred, Hungary, 2012, pp. 243-246.

[14] Rasovic, N.; Kaljun, J.; Obad, M.; Novak, M.; Dolšak, B. The CAD models evaluation using weighting factors in order to optimize RPT process in Advanced Engineering, Computer Aided Design and Manufacturing CADAM 2012, Vis, Croatia, 2012, pp. 95-98. $\begin{array}{llcc}\text { [15] Industrial } & 3 \mathrm{D} & \text { Measurement } & \text { Technology. } \\ \text { polska.com.pl/images/GOM/Katalogi/katalog2.pdfJ. Van der Geer, J.A.J. }\end{array}$ writing a scientific article, J. Sci. Commun. 163 (2000), pp. 51-59.

Available: $\quad$ http://www.itaHanraads, R.A. Lupton, The art of 